\title{
THE RELIABILITY OF MEASUREMENTS OF PELVIC RADIOGRAPHS IN INFANTS
}

\author{
F. G. BONIFORTI, G. FUJII, R. D. ANGLISS, M. K. D. BENSON \\ From the Nuffield Orthopaedic Centre, Oxford, England
}

We have evaluated the reliability of the measurement of radiological indicators in developmental dysplasia of the hip. Three observers each independently assessed 60 pelvic radiographs from infants aged from 3 to 36 months. Errors from the true value of a single measurement made by a single observer (E1), of the average of two measurements by a single observer (E2), and of the average of two single measurements by two different observers (E3) were established for the acetabular index of Hilgenreiner, for the assessment of superior and lateral femoral displacement and for indicators of pelvic alignment.

The errors for the assessment of the acetabular index were $\mathrm{E} 1 \pm 5^{\circ}, \mathrm{E} 2 \pm 5^{\circ}$, and $\mathrm{E3} \pm 3.5^{\circ}$. There was a significant correlation between the presence of an acetabular notch on the radiograph and an increased error in measurement $(\mathbf{p}=\mathbf{0 . 0 1})$. Yamamuro's measurement of lateral femoral displacement was more reliable than the Hilgenreiner distance. The errors of indicators of pelvic alignment showed a correlation with the age of the infant; the quotient of pelvic rotation was more reliable after seven months of age $(p<0.0001)$. The errors of the measurement of the symphysis os-ischium angle tended to increase with age and those of the measurement of the index of pelvic tilt decreased with skeletal maturation $(\mathbf{p}=\mathbf{0 . 0 0 2})$.

J Bone Joint Surg [Br] 1997;79-B:570-5.

Received 9 September 1996; Accepted after revision 18 February 1997

F. G. Boniforti, MD, Orthopaedic Registrar

Clinica Ortopedica I, Universita degli Studi di Milano, P. Cardinal Ferrari 1, 20122 Milano, Italy.

G. Fujii, MD, PhD, Senior Lecturer

Department of Orthopaedic Surgery, Tohoku University School of Medicine, 1-1 Seiryo-cho, Aoba-Ku, Sendai 980, Japan.

R. D. Angliss, FRACS, Clinical Fellow

M. K. D. Benson, FRCS, Consultant Orthopaedic Surgeon

Nuffield Orthopaedic Centre NHS Trust, Windmill Road, Headington, Oxford OX3 7LD, UK.

Correspondence should be sent to Mr M. K. D. Benson.

(C)1997 British Editorial Society of Bone and Joint Surgery 0301-620X/97/47238\$2.00
The diagnosis and assessment of developmental dysplasia of the hip (DDH) rely greatly on radiological interpretation and measurements of plain radiographs are widely accepted. ${ }^{1}$ Various indicators have been introduced to evaluate severity and response to treatment; normal values have been established. $^{2-6}$ These studies were based on single observations made by single observers, and there are few assessments of the reliability of such measurements. ${ }^{7,8}$

We studied the reliability of some common radiological measurements used for the diagnosis and follow-up of DDH in infants.

\section{MATERIAL AND METHODS}

We studied 60 anteroposterior pelvic radiographs of infants aged between 3 and 36 months, all taken in the radiology department of the Nuffield Orthopaedic Centre, Oxford. Thirty radiographs were of infants from a 'normal-at-risk' population, taken as part of a screening programme and 30 were of infants with established DDH. Ten radiographs from each group were in each of three age categories: 3 to 6 months, 7 to 12 months, and 13 to 36 months. We then measured the angles, lines, and other indicators used for the diagnosis of DDH.

\section{Measurement of angles}

Acetabular index. This was measured as the angle between the Y-line (connecting the lowest points of the ilium) and the line which connects the lowest point of the ilium and the acetabular edge (Fig. 1). ${ }^{2}$

Acetabular notch. The radiological appearance of the acetabular notch ${ }^{9}$ was recorded for all radiographs. It was seen as a scooped deformity on the superolateral aspect of the acetabular edge (see Fig. 5); this edge was then identified as the superolateral point of the notch (Fig. 1).

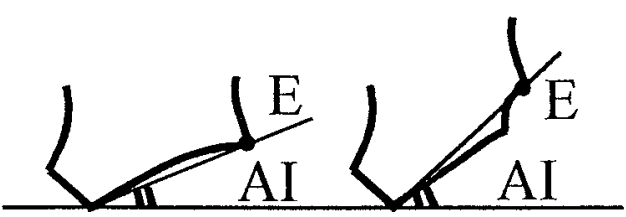

Fig. 1

Diagram showing the acetabular edge (E) with and without the notch (AI; acetabular index).

THE JOURNAL OF BONE AND JOINT SURGERY 

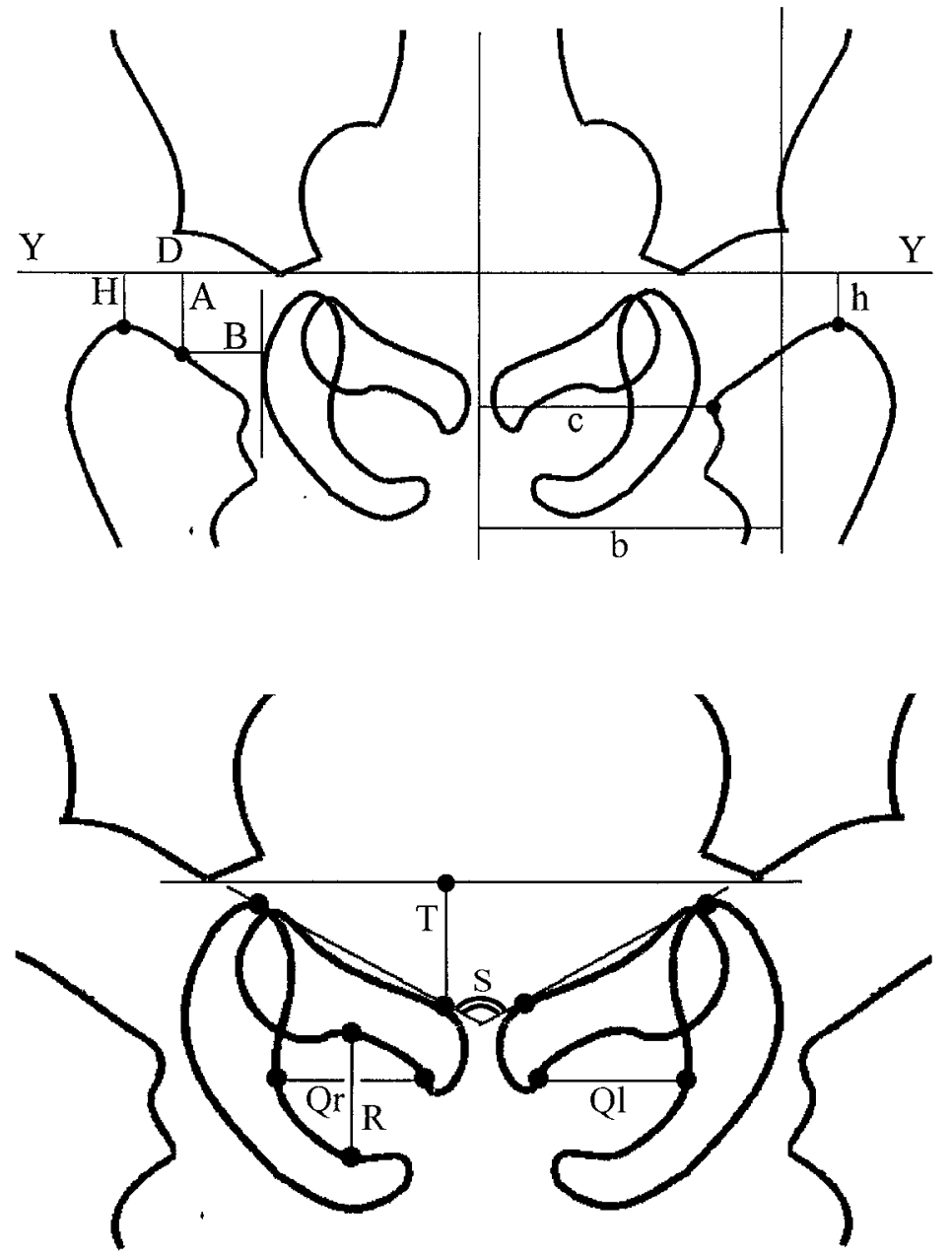

Fig. 2

Diagram of the radiological appearance of the infant pelvis with femoral displacement parameters (Y, Y-line; A, Yamamuro-A; B, Yamamuro-B; H, Hilgenreiner-H; $\mathrm{D}$, Hilgenreiner-D; $\mathrm{h} / \mathrm{b}$ and $\mathrm{c} / \mathrm{b}$ ratio, Smith's ratio for superior and lateral displacement).
Fig. 3

Diagram showing the quotient of pelvic rotation (Qr/ Ql) the symphysis os-ischium angle (S), and the pelvic tilt index $(R / T)$.
Linear measurements of superior femoral displacement Yamamuro-A distance. ${ }^{5}$ This is the distance in millimetres between the middle point of the proximal femoral metaphysis and the Y-line ('A' in Fig. 2). The range of normal values for infants of one month to four years of age is 7 to $14 \mathrm{~mm}$.

Hilgenreiner-H distance. ${ }^{2}$ This is the distance between the highest point of the proximal femoral metaphysis and the Y-line (' $\mathrm{H}$ ' in Fig. 2). The normal value is 8 to $10 \mathrm{~mm}$. $h / b$ ratio. ${ }^{4}$ This is the ratio of the distance between the highest point of the femoral metaphysis and the Y-line (' $h$ ' in Fig. 2), and the distance between Perkins' line and a parallel line passing through the centre of the sacrum (centre line: 'b' in Fig. 2). The normal value for infants of two to five years of age is 0.10 to 0.20 .

Linear measurements of lateral femoral displacement Yamamuro- $B$ distance. ${ }^{5}$ This is the distance in millimetres between the middle point of the proximal femoral metaphysis and a line, perpendicular to the Y-line, which passes through the lateral edge of the ischium ('B' in Fig. 2). The normal value for children of one month to four years of age is 5 to $12 \mathrm{~mm}$.
Hilgenreiner-D distance. ${ }^{2}$ This is the distance in millimetres between the inferior bony margin of the ilium and the projection on the Y-line of the highest point of the proximal femoral metaphysis (' $D$ ' in Fig. 2). The normal value is 14 to $16 \mathrm{~mm}$.

$c / b$ ratio. ${ }^{4}$ This is the ratio of the distance between the medial beak of the proximal femoral metaphysis and the centre line ('c' in Fig. 2), and the distance between Perkins' line and the centre line ('b' in Fig. 2). The normal value for infants of two to five years of age is 0.60 to 0.85 .

Indicators of pelvic alignment

Quotient of pelvic rotation. ${ }^{6}$ This evaluates the pelvic position in the horizontal plane. It is the ratio of the horizontal diameter of the obturator foramen of the right side and that of the left ('Qr' and 'Ql' in Fig. 3). In neutral rotation the ratio is 1 , but is considered to be acceptable when it is between 0.56 and 1.8 .

Symphysis os-ischium angle. ${ }^{6}$ This evaluates the pelvic position in the sagittal plane. Tönnis ${ }^{10}$ described the angle formed "by two lines which are tangential to the highest point on each ischium and which meet at the point of the symphysis that projects farthest into the pelvic aperture" 
Table I. Reliability of radiological indicators

\begin{tabular}{lllll}
\hline Indicator & $\begin{array}{l}\text { Range of } \\
\text { observation }\end{array}$ & E1* & E2* & E3* \\
\hline Acetabular index (degrees) & 13 to 47 & \pm 5 & \pm 5 & \pm 3.5 \\
Yamamuro-A (mm) & 5 to 17 & \pm 1.9 & \pm 1.8 & \pm 1.3 \\
Hilgenreiner-H (mm) & 2 to 15 & \pm 1.9 & \pm 1.9 & \pm 1.4 \\
h/b ratio & 0.04 to 0.33 & \pm 0.04 & \pm 0.04 & \pm 0.03 \\
Yamamuro-B (mm) & 4 to 14 & \pm 1.8 & \pm 1.7 & \pm 1.3 \\
Hilgenreiner-D (mm) & 14 to 28 & \pm 2.9 & \pm 2.7 & \pm 2 \\
c/b ratio & 0.69 to 0.9 & \pm 0.05 & \pm 0.05 & \pm 0.04 \\
Quotient of pelvic rotation (ratio) & 0.6 to 1.8 & \pm 0.19 & \pm 0.19 & \pm 0.13 \\
Symphysis os-ischium angle (degrees) & 86 to 128 & \pm 6.7 & \pm 6.4 & \pm 4.7 \\
Pelvic tilt index (ratio) & 0.4 to 1.6 & \pm 0.17 & \pm 0.14 & \pm 0.12 \\
\hline * see text & & & &
\end{tabular}

(' $\mathrm{S}$ ' in Fig. 3). The range of normal values is from 90 to $135^{\circ}$ and is related to the infant's age.

Pelvic tilt index. ${ }^{3}$ This also assesses the pelvic position in the sagittal plane and is the ratio between the vertical diameter of the obturator foramen and the distance between the upper brim of the pubis and the Y-line (' $R$ ' and ' $T$ ' in Fig. 3). With the pelvis normally positioned the ratio is between 0.75 and 1.2 .

\section{Method of assessment}

To determine the reliability of the measurements, we calculated variances and errors for all parameters. The observers first agreed on the precise definition of landmarks to be used $^{11}$ and each then measured all the radiographs on two occasions one week apart. Landmarks were marked on the radiographs by small crosses using sharp soft lead pencils. Each observer then used a graphic digitiser linked to a personal computer (KL-4300 Graphtec Japan, Tokyo, Japan) to record the position. A computer program written by one author (GF) on N88BASIC/MS-DOS ver 5.0 (NEC, Tokyo, Japan) then calculated the values of the measured parameters. Each observer rubbed out the marks on each radiograph after digitalisation.

Comparison between groups. The variances of all parameters were compared between the 'normal-at risk' and the $\mathrm{DDH}$ groups to determine how much variability of the measurements was induced by the dysplastic hip.

We compared the variances of the acetabular index between the groups with and without the radiological appearance of an acetabular notch to assess the relation of reliability to the shape of the acetabular edge. The variances of indicators of pelvic alignment were determined for each of the three age groups to ascertain whether growth affected the reliability of measurement.

Statistical methods. We assessed reliability by partitioning the variance as described by Streiner and Norman. ${ }^{12}$ Estimations were made of intraobserver variance, variance due to interaction between observers and measurements, and variance due to different observers (interobserver). From these estimates a range within which the parameters of the true value could be estimated with $95 \%$ confidence was calculated for three situations:
E1: a single measurement by a single observer.

E2: the average of two measurements by a single observer.

E3: The average of two single measurements from two different observers.

Comparison between groups was calculated by the ratio of variances using the F-test.

\section{RESULTS}

Errors (E1, E2 and E3) were calculated for all the parameters (Table I).

Acetabular index. Errors for the acetabular index for the total patient group were $\mathrm{E} 1 \pm 5^{\circ}, \mathrm{E} 2 \pm 5^{\circ}, \mathrm{E} 3 \pm 3.5^{\circ}$ (range 13 to 47; Table I). For the normal-at-risk group they were $\mathrm{E} 1 \pm 4.3^{\circ}, \mathrm{E} 2 \pm 4.3^{\circ}, \mathrm{E} 3 \pm 3^{\circ}$ and for the DDH group E1 \pm $5.7^{\circ}, \mathrm{E} 2 \pm 5.6^{\circ}, \mathrm{E} 3 \pm 4^{\circ}$ (Table II). A comparison between the normal-at risk and the DDH groups showed significant differences $(p=0.02)$. Of the 120 hips investigated, 48 had radiological evidence of an acetabular notch. For the hips without the notch the errors were E1 $\pm 4.5^{\circ}$, E2 $\pm 4.5^{\circ}, \mathrm{E} 3$ $\pm 3.1^{\circ}$ and for those with the notch $\mathrm{E} 1 \pm 6.1^{\circ}, \mathrm{E} 2 \pm 5.9^{\circ}, \mathrm{E} 3$ $\pm 4.3^{\circ}$ (Table II). The acetabular index variances between the groups with and without the notch were significantly different $(p=0.01)$, showing that a deformed acetabular edge increases the error of measurement of the acetabular index.

Linear measurements of superior femoral displacement Yamamuro-A distance. The errors were E1 $\pm 1.9 \mathrm{~mm}, \mathrm{E} 2 \pm$ $1.8 \mathrm{~mm}, \mathrm{E} 3 \pm 1.3 \mathrm{~mm}$ (range 5 to 17 ; Table I).

Hilgenreiner- $H$ distance. The errors were E1 $\pm 1.9 \mathrm{~mm}, \mathrm{E} 2$ $\pm 1.9 \mathrm{~mm}$, E3 $\pm 1.4 \mathrm{~mm}$ (range 2 to 15 ; Table I).

$h / b$ ratio. The errors were $\mathrm{E} 1 \pm 0.04, \mathrm{E} 2 \pm 0.04, \mathrm{E} 3 \pm 0.03$ (range 0.033 to 0.04 ; Table I).

Linear measurements of lateral femoral displacement Yamamuro- $B$ distance. The errors were E1 $\pm 1.8 \mathrm{~mm}, \mathrm{E} 2 \pm$ $1.7 \mathrm{~mm}, \mathrm{E} 3+1.3 \mathrm{~mm}$ (range 4 to 14 ; Table I).

Hilgenreiner $-D$ distance. The errors were E1 $\pm 2.9 \mathrm{~mm}, \mathrm{E} 2$ $\pm 2.7 \mathrm{~mm}$, E3 $\pm 2 \mathrm{~mm}$ (range 14 to 28 ; Table I).

$c / b$ ratio. The errors were E1 $\pm 0.05, \mathrm{E} 2 \pm 0.05, \mathrm{E} 3 \pm 0.04$ (range 0.69 to 0.9 ; Table I). 
Table II. Reliability according to groups

\begin{tabular}{lllll}
\hline Indicator & Number & E1* & E2* & E3* \\
\hline Acetabular index (degrees) & & & & \\
$\quad$ Normal-at risk & 60 & \pm 4.3 & \pm 4.3 & \pm 3 \\
$\quad$ DDH & 80 & \pm 5.7 & \pm 5.6 & \pm 4 \\
$\quad$ Without notch & 48 & \pm 4.5 & \pm 4.5 & \pm 3.1 \\
$\quad$ With notch & & & & \\
Quotient of pelvic rotation (ratio) & 20 & \pm 0.27 & \pm 0.27 & \pm 0.19 \\
3 to 6 months & 20 & \pm 0.14 & \pm 0.13 & \pm 0.1 \\
7 to 12 months & 20 & \pm 0.1 & \pm 0.1 & \pm 0.07 \\
13 to 36 months & & & & \\
Symphysis os-ischium angle (degrees) & 20 & \pm 5.6 & \pm 5.5 & \pm 3.9 \\
3 to 6 months & 20 & \pm 6.3 & \pm 5.8 & \pm 4.4 \\
7 to 12 months & 20 & \pm 7.3 & \pm 7.3 & \pm 5.2 \\
13 to 36 months & & & & \\
Pelvic tilt index (ratio) & 40 & \pm 0.17 & \pm 0.15 & \pm 0.12 \\
3 to 6 months & 40 & \pm 0.12 & \pm 0.11 & \pm 0.09 \\
7 to 12 months & 40 & \pm 0.1 & \pm 0.1 & \pm 0.07 \\
\hline 13 to 36 months & & & &
\end{tabular}

\section{Indicators of pelvic alignment}

Quotient of pelvic rotation. The errors were E1 \pm 0.19 , E2 \pm 0.19 , E3 \pm 0.13 (range 0.6 to 1.8 ; Table I). For the 3- to 6 -month age group they were $\mathrm{E} 1 \pm 0.27, \mathrm{E} 2 \pm 0.27, \mathrm{E} 3 \pm$ 0.19 ; for the 7- to 12 -month age group $\mathrm{E} 1 \pm 0.14$, E2 \pm 0.13 , E3 + 0.1; and for the 13- to 36-month age group E1 \pm $0.1, \mathrm{E} 2 \pm 0.1, \mathrm{E} 3 \pm 0.07$ (Table II). Comparison between the variances of the first age group ( 3 to 6 months) and the other two were significantly different $(\mathrm{p}<0.0001)$.

Symphysis os-ischium angle. The errors were $\mathrm{E} 1 \pm 6.7^{\circ}, \mathrm{E} 2$ $\pm 6.4^{\circ}$, E3 $\pm 4.7^{\circ}$ (range 86 to 128 ; Table I). For the 3- to 6 -month age group they were $\mathrm{E} 1 \pm 5.6^{\circ}, \mathrm{E} 2 \pm 5.5^{\circ}, \mathrm{E} 3 \pm$ $3.9^{\circ}$, for the 7- to 12 -month age group E1 $\pm 6.3^{\circ}, \mathrm{E} 2 \pm 5.8^{\circ}$, $\mathrm{E} 3 \pm 4.4^{\circ}$ and for the 13 - to 36 -month age group E1 $\pm 7.3^{\circ}$, $\mathrm{E} 2 \pm 7.3^{\circ}, \mathrm{E} 3 \pm 5.2^{\circ}$ (Table II).

Pelvic tilt index. The errors were E1 $\pm 0.17, \mathrm{E} 2 \pm 0.14$, E3 \pm 0.12 (range 0.4 to 1.6; Table I). For the 3- to 6-month age group they were $\mathrm{E} 1 \pm 0.17, \mathrm{E} 2 \pm 0.15, \mathrm{E} 3 \pm 0.12$, for the 7 to 12 -month age group $\mathrm{E} 1 \pm 0.12, \mathrm{E} 2 \pm 0.11, \mathrm{E} 3 \pm 0.09$, and for the 13- to 36-month age group E1 \pm 0.1 , E2 \pm 0.1 , E3 \pm 0.07 (Table II). The difference between the 3- to 6-month and the 13- to 36-month age groups was significant $(\mathrm{p}=0.002)$.

Except for the acetabular index, we found no significant differences in variances of the radiological indicators between the normal-at-risk and DDH groups.

\section{DISCUSSION}

The radiological appearance of the pelvis varies widely in early infancy when the whole femoral head and most of the acetabulum consist of cartilage (Figs 4 and 5). A precise analysis of the radiological appearance of the hip is needed to assess DDH in infancy. Our study suggests that there are differences in measurements on infant pelvic radiographs recorded by the same observer on two occasions and by different observers. These are most marked in deformities of the acetabular margin and in different skeletal immaturities.

The acetabular index of Hilgenreiner is widely used, and the normal range has been defined. ${ }^{10,13}$ Our results suggest that variability in its measurement in DDH must be recognised, particularly in dysplastic hips with an acetabular notch. If measurement of the acetabular index is to be used for prognosis during treatment of $\mathrm{DDH},{ }^{13}$ precise definition of the landmarks is essential to allow sequential radiographs to be compared and analysed.

Measurements of femoral displacement are useful in evaluating the relationship between the femur and the acetabulum. The bony shape of the hip may vary widely on radiographs of the infant pelvis. Wilkinson ${ }^{14}$ noted that the proximal femoral metaphysis is often rounded, with no well-demarcated border (Figs 4 and 5); in such circumstances it may be difficult to identify the highest point of the proximal femoral metaphysis. The position of the limb will also influence the measurable distance between the highest point of the metaphysis and the Y-line more than that between the mid-point of the metaphysis and the Yline. ${ }^{5}$ For these reasons we prefer to use Yamamuro's method of assessing femoral displacement.

Smith's ratios are reliable. The use of a ratio for radiological measurement compared with measurement of a direct distance reduces the influence of radiological magnification. The use of two distances for the ratio involves at least four landmarks instead of two and may decrease accuracy. Several authors have assessed the error measurement induced by the position of the infant pelvis. ${ }^{3,6,8}$ Indicators of pelvic alignment were introduced to establish the radiological orientation of the pelvis in both horizontal and sagittal planes in order to ensure adequate symmetry; ${ }^{3,10}$ their reliability correlates well with age. Indicators such as the quotient of pelvic rotation and the pelvic tilt index, which use the obturator foramen as a landmark, are less accurate before the ossification of 


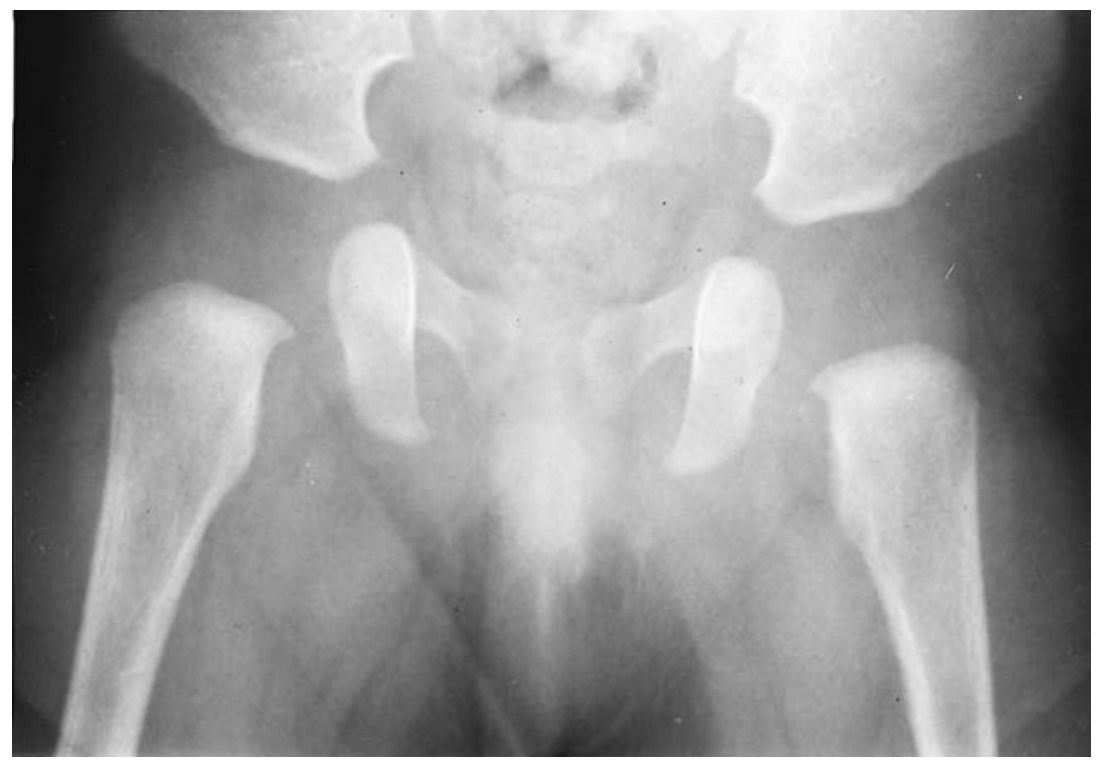

Fig. 4

Radiograph of the pelvis of a five-month-old infant showing the triangular shape of the obturator foramen, the highest point of the ischium, and the rounded proximal femoral metaphysis.

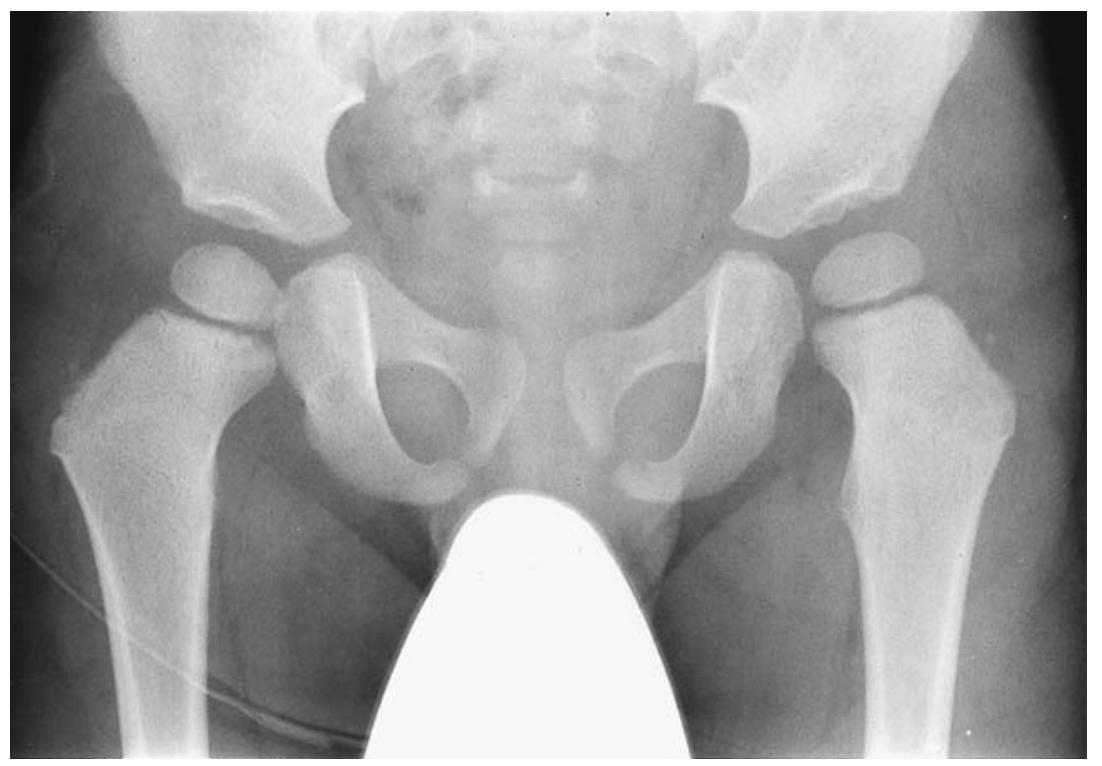

Fig. 5

Radiograph of the pelvis of a 27-month-old infant showing an acetabular notch on the left side, the ovoid shape of the obturator foramen, the overlapped highest point of the ischium, and the sharp proximal femoral metaphysis. the ischiopubic synchondrosis. With growth of the pelvis the radiological shape of the obturator foramen changes from nearly triangular to ovoid ${ }^{15}$ and its horizontal and vertical diameters become more definite (Figs 4 and 5); the reliability of the quotient of pelvic rotation and the pelvic tilt index then increases. The symphysis osischium angle uses the superior edge of the ischium as a landmark. With progressive growth and ossification of the triradiate cartilage the superior edge of the ischium overlaps the upper part of the superior pubic ramus ${ }^{16}$ and reduces the reliability of this landmark, which, in turn, decreases the accuracy of the measurements of the symphysis os-ischium angle.

Radiological parameters are extremely useful in the diagnosis and management of DDH, but the reliability of landmarks and measurements cannot be assumed; appro- priate care must be taken in judging appearances. The acetabular index should be used only after the observer has clearly established the proper landmarks for the acetabulum. Yamamuro's measurements for linear displacement of the femoral head are accurate and less influenced by femoral rotation. The quotient of pelvic rotation becomes more accurate after seven months of age. The symphysis os-ischium angle is useful up to the second year of life, but after this the pelvic tilt index becomes more reliable. It is best to use the indicators of pelvic alignment which are most appropriate to the age of the infant.

We wish to thank Ms Angie Wade (the Institute of Child Health, London) for her help and advice in statistical analysis.

No benefits in any form have been received or will be received from a commercial party related directly or indirectly to the subject of this article. 


\section{REFERENCES}

1. Catterall A. Editorial. The early diagnosis of congenital dislocation of the hip. J Bone Joint Surg [Br] 1994;76-B:515-6.

2. Hilgenreiner $\mathbf{H}$. Early diagnosis and early treatment of congenital dislocation of the hip. Med Klin 1925;21:1385-8;1425-9.

3. Ball F, Kommenda K. Sources of error in the roentgen evaluation of the hip in infancy. Ann Radiol (Paris) 1968;11:298-303.

4. Smith WS, Badgley CE, Orwig JB, Harper JM. Correlation of postreduction roentgenograms and thirty-one-year follow-up in congenital dislocation of the hip. J Bone Joint Surg [Am] 1968;50-A: 1081-98.

5. Yamamuro T, Chene S-H. A radiological study on the development of the hip joint in normal infants. J Jap Orthop Ass 1975;49:421-39.

6. Tönnis D. Normal values of the hip joint for the evaluation of $\mathrm{x}$-rays in children and adults. Clin Orthop 1976;119:39-47.

7. Broughton NS, Brougham DI, Cole WG, Menelaus MB. Reliability of radiological measurements in the assessment of the child's hip. $J$ Bone Joint Surg [Br] 1989;71-B:6-8.

8. Portinaro NM, Murray DW, Bhullar TP, Benson MK. Errors in measurement of acetabular index. J Pediatr Orthop 1995;15:780-4.
9. Portinaro NMA, Matthews SJE, Benson MKD. The acetabular notch in hip dysplasia. J Bone Joint Surg [Br] 1994;76-B:271-3.

10. Tönnis D. Congenital dysplasia and dislocation of the hip in children and adults. Berlin, etc: Springer-Verlag, 1987.

11. Katz JF. Precise identification of radiographic acetabular landmarks. Clin Orthop 1979;141:166-8.

12. Streiner DL, Norman GR. Health measurement scales. 2nd ed. Oxford, etc: Oxford University Press, 1995:79-105.

13. Tasnavites A, Murray DW, Benson MKD. Improvement in acetabular index after reduction of hips with developmental dysplasia. $J$ Bone Joint Surg [Br] 1993;75-B:755-9.

14. Wilkinson JA. Congenital dislocation of the hip joint. New York, Springer, 1985.

15. Köhler A, Zimmer EA. Wilk SP, translator and ed. Borderlands of the normal and early pathologic in skeletal roentgenology. 3rd ed based on 11th ed. New York, etc: Grune \& Stratton, 1968.

16. Plaster RL, Schoenecker PL, Capelli AM. Premature closure of the triradiate cartilage: a potential complication of pericapsular acetabuloplasty. J Pediatr Orthop 1991;11:676-8. 